\title{
Functional MRI of Postnatal Visual Development in Normal Rat Superior Colliculi
}

\author{
Kevin C. Chan, Kai Xing, Matthew M. Cheung, Iris Y. Zhou, and Ed X. Wu ${ }^{*}$
}

\begin{abstract}
This study employed blood oxygenation leveldependent functional MRI (BOLD-fMRI) to evaluate the visual responses in the superior colliculus of the developing rat brain from the time of eyelid opening to adulthood. Upon flash illumination to the contralateral eye, the regional BOLD response underwent a systematic increase in amplitude with age especially after the third postnatal week. However, no significant difference in BOLD signal increase was found between postnatal days 14 and 21 . Our results constitute the first fMRI report in demonstrating the critical period of visual functions in the rat brain during maturation. This can be potentially useful in establishing the links between changes in relation to visual sensory development.
\end{abstract}

\section{INTRODUCTION}

$\mathrm{F}$ unctional magnetic resonance imaging (fMRI) techniques provide the capability for visualizing dynamics of neural activities with spatiotemporal specificity and resolution that has not previously been available with other non-invasive methods, and are of great potentials for studying early brain development. Recent fMRI studies have demonstrated their values for probing postnatal circuit refinement and plasticity [1-3]. Yet, to date, many aspects of brain development are required to be further explored, such as neurosensory and neuromotor development, development of cognition with learning processes, memory and language acquisition, as well as brain repair and plasticity after perinatal injury [2, 4]. In this study, the development of visual activations in the rat visual pathway was studied using blood oxygenation level-dependent (BOLD) contrast from the time of eyelid opening at about postnatal day (P) 14 to adulthood at P60. By studying BOLD-fMRI measurements in the rat superior colliculus, we determined that the regional BOLD response in these animals underwent a systematic increase in amplitude

Manuscript received April 7, 2009. This work was supported in part by the Hong Kong Research Grant Council and The University of Hong Kong CRCG grant.

Kevin C. Chan, Kyle K. Xing, Matthew M. Cheung, and Iris Y. Zhou are with the Laboratory of Biomedical Imaging and Signal Processing and the Department of Electrical and Electronic Engineering, The University of Hong Kong, Hong Kong SAR, China (e-mail: kevin_ccw@hkuusa.hku.hk, xingkai@eee.hku.hk, thew@hkusua.hku.hk, iriszhou@eee.hku.hk)

Ed X. Wu is with the Laboratory of Biomedical Imaging and Signal Processing and the Department of Electrical and Electronic Engineering, The University of Hong Kong, Hong Kong SAR, China (corresponding author to provide phone: (852) 2819-9713; fax: (852) 2819-9711; e-mail: ewu@eee.hku.hk). after the third postnatal week. The results presented here are potentially useful to the in vivo and longitudinal study of functional activities in developing brains under normal and pathological conditions and in therapeutic interventions.

\section{MATERIALS AND METHODS}

\section{A. Animal Preparation}

Pregnant Sprague-Dawley rats were obtained approximately 2 days before parturition, and their litters were culled to 9 to 13 pups. Neonate rats were kept with their mother in regular light/dark cycle for 7 days after birth. A total of 7 P7 rats, ranging from 12 to $16 \mathrm{~g}$ in weight, were randomly selected for MRI scans, from the time of eyelid opening at about P14, to P21, P28 and adulthood at P60. At P21, the pups were separated from their mother and were reared in a temperature-controlled room subjected to a $12 \mathrm{~h}$ light/dark cycle with standard chow and water supply ad libitum. $\mathrm{T}_{1}$-weighted imaging (T1WI), $\mathrm{T}_{2}$-weighted imaging (T2WI) and BOLD-fMRI were performed to all animals.

\section{B. MRI Protocols}

All MRI measurements were acquired utilizing a 7 Tesla Bruker scanner. Under inhaled isoflurane anaesthesia $(3 \%$ induction and $1 \%$ maintenance), animals were kept warm under circulating water at $37^{\circ} \mathrm{C}$ and were imaged using a receive-only surface coil. Continuous monitoring of the respiration rate was performed and maintained within normal physiological ranges. Scout $\mathrm{T} 2 \mathrm{~W}$ images were first acquired in three planes with a rapid acquisition with relaxation enhancement (RARE) pulse sequence to position the subsequent multi-parametric MR images along standard anatomical orientations in a reproducible manner. High-resolution 2D T1W RARE pulse sequence was then acquired at the level of the superior colliculi with field of view $($ FOV $)=32 \times 24 \mathrm{~mm}^{2}$, matrix resolution $=256 \times 192$, slice thickness $=1 \mathrm{~mm}$, number of slices $=6$, repetition time/echo time $(\mathrm{TR} / \mathrm{TE})=400 / 7.5 \mathrm{~ms}$, RARE factor $=4$ and number of averages $=16$; T2WI was performed under the same spatial dimensions, with TR/TE $=4200 / 38.7 \mathrm{~ms}$, RARE factor $=8$ and number of averages $=2$; For BOLD-fMRI, single-shot, spin-echo echo planar imaging sequence (SE-EPI) was acquired with TR/TE $=2000 / 21 \mathrm{~ms}, \mathrm{FOV}=3.2 \times 2.4 \mathrm{~cm}^{2}$, 
matrix resolution $=64 \times 48$ (zero-filled to $64 \times 64$ ), slice thickness $=1 \mathrm{~mm}$, number of slices $=6$, number of time points $=110$ and total acquisition time $=3$ mins $40 \mathrm{~s}$.

\section{Stimulation Paradigm}

Two fiber optic cables, each with a green light-emitting diode (LED) at one end, were placed bilaterally at about $1 \mathrm{~cm}$ away from the eyes of the rats. The LEDs were flashed at a frequency of $1 \mathrm{~Hz}$ and a pulse width of $50 \mathrm{~ms}$. A standard block-design visual stimulation protocol of $40 \mathrm{~s}$ of rest followed by stimulation for $20 \mathrm{~s}$ repeated for 3 blocks was used under computerized control using LabVIEW v8.0 (National Instruments Corporation, Austin, TX). The rats were allowed to rest for few minutes between stimulation sets, and 2-5 sets of data were recorded from each eye for each rat.

\section{Data Analysis}

All fMRI data were co-registered using SPM5 (Statistical Parametric Mapping, Wellcome Department of Imaging Neuroscience, Functional Imaging Laboratory, London, UK). Model-driven cross-correlation analysis was performed using the STIMULATE software package (Stimulate, Center for Magnetic Resonance Research, University of Minnesota) with a correlation threshold of 0.15 and a cluster of 3 pixels. Time profiles of BOLD signals were collected and temporally low-pass filtered from the activated superior colliculi contralateral to the stimulated eye. Percentage changes of BOLD signals were calculated and averaged among animals from the same age groups. All data were presented as mean \pm standard error of mean (S.E.M.). Mean values were compared using two-tailed Student's t-tests between left and right superior colliculi upon contralateral eye stimulation, and between age groups on the same side of eye stimulation. Results were considered to be significantly different when $\mathrm{p}<0.05$.

\section{RESULTS}

\section{A. General Pattern of BOLD Responses in Adult Rat Primary Visual System}

The patterns of visual responses in the adult P60 rat brain generally followed as previously described [5, 6]. In particular, activations by flash to either eye showed that responses were localized mainly to the contralateral superior colliculus, and sometimes the contralateral and ipsilateral visual cortex (Fig. 1). In the BOLD response dynamic profile, a hemodynamic delay in the order of seconds was found after initial stimulation before reaching the peak height (Fig. 2, green curve). No significant difference in the BOLD percentage increase was observed between the left and right superior colliculi upon contralateral eye stimulation ( $\mathrm{p}=0.32)$.

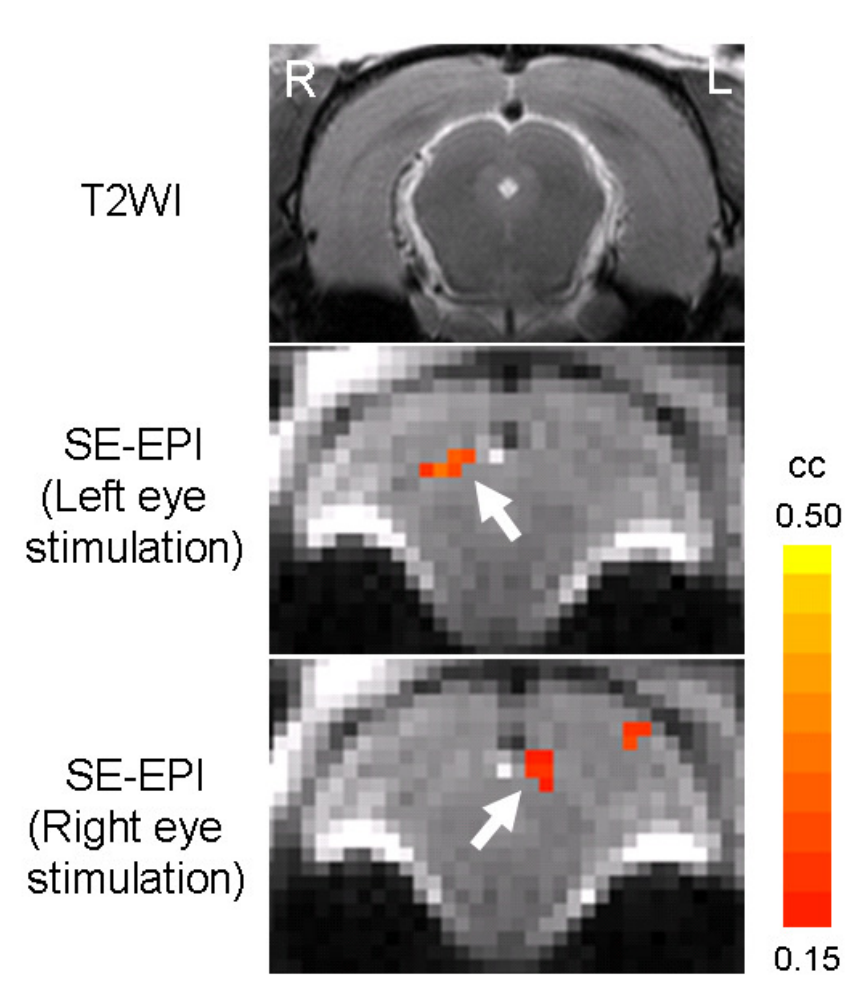

Fig 1: Typical T2WI (top), and SE-EPI (middle and bottom) of a normal P60 brain at the level of the superior colliculi. Upon left (middle) and right (bottom) eye stimulations, activations were observed in the contralateral superior colliculus (arrows) in the cross correlation maps overlaid on the SE-EPI.

\section{B. Functional Development of Normal Superior Colliculi}

In the current study, the 7 neonatal rats had their eyelids open at P14.5 \pm 0.2 . As shown in Fig. 2, qualitative evaluation showed that the averaged BOLD time courses had a trend of increasing peak amplitude of the activated BOLD signals as the rat grew. In particular, the responsiveness enhanced markedly with age beyond the third postnatal week at P28 and P60, but less between P14 and P21. Note also the higher rate of BOLD signal increase after initial stimulation at P60 compared to the other age groups. Quantitatively, upon right eye stimulation, the averaged stimulus-induced BOLD percentage signal changes in the left superior colliculus were $0.44 \pm 0.18 \%, 0.63 \pm 0.25 \%, 0.92 \pm 0.14 \%$, and $1.46 \pm 0.16 \%$ at P14, P21, P28 and P60, respectively; whereas upon left eye stimulation the BOLD signal changes were $0.53 \pm 0.14 \%$, $0.62 \pm 0.32 \%, 0.90 \pm 0.15 \%$, and $1.43 \pm 0.19 \%$, respectively, in the right superior colliculus. Significant differences in BOLD signal increase on either side of the SC were observed between P14 and P28, between P21 and P28, and between $\mathrm{P} 60$ and each of the 3 other age groups $(\mathrm{p}<0.05)$. No significant difference in the BOLD percentage increase was observed between P14 and P21, or between the left and right superior colliculi upon contralateral eye stimulation in any age groups ( $\mathrm{p}>0.05$ ) (Fig. 3). 


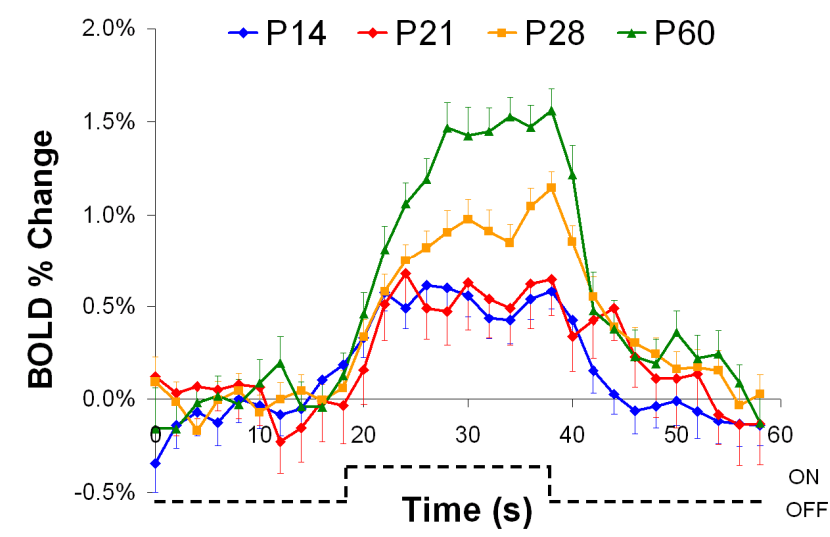

Fig. 2. Averaged dynamic profiles of BOLD responses to visual stimulation in the superior colliculi of normal rats at P14, P21, P28 and P60. The time courses were computed from the activated voxels in the superior colliculus contralateral to the stimulated eye, which were then averaged across all successful trials in each age group. Note that increasing age appeared to associate with a higher BOLD signal increase in the stimulation 'on' period after the third postnatal week at P28 and P60, but less between P14 and P21.

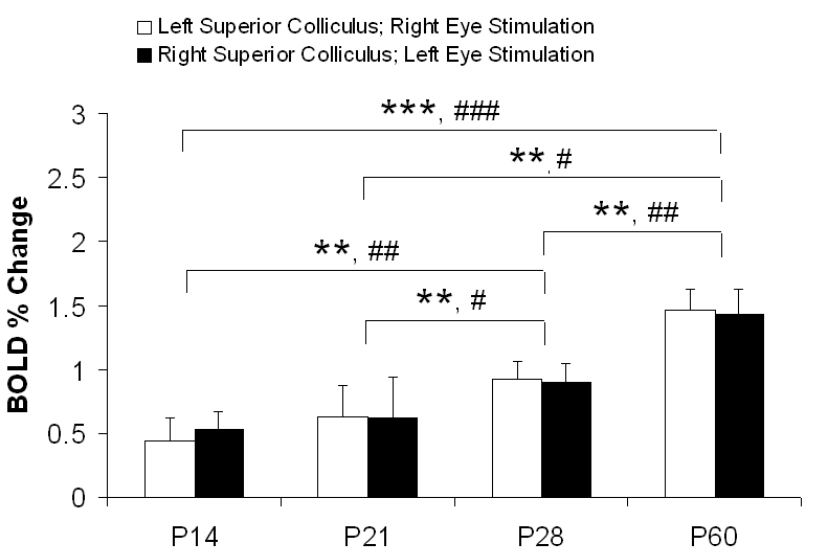

Fig. 3. Age-dependent changes in BOLD-fMRI responses to visual stimulation in normal rat brains from P14 to P60. Significant differences in BOLD signal increase on either side of the SC were observed between P14 and P28, between P21 and P28, and between P60 and each of the 3 other age groups $(\mathrm{p}<0.05)$. No significant differences in BOLD percentage increase were observed between $\mathrm{P} 14$ and P21, or between the left and right superior colliculi upon contralateral eye stimulation in all age groups $(p>0.05)$. $(* \mathrm{p}<0.05, * * \mathrm{p}<0.01$ and $* * * \mathrm{p}<0.001$ between age groups in the left $\mathrm{SC}$; $\# p<0.05, \# \# p<0.01$ and $\# \# \# p<0.001$ between age groups in the right SC.)

\section{DISCUSSIONS}

Our results constitute the first fMRI report in evaluating the visual development of the rat superior colliculus from the time of eyelid opening (P14) to adulthood (P60). Similar to the development of hemodynamic responses in rat somatosensory cortex [1], age increase was associated with an increase in BOLD signal amplitude in the rat superior colliculus. However, while the rat somatosensory cortex underwent a systematic growth in amplitude from P13-15 to P20-30 to adulthood [1], the visual function in the superior colliculus appeared to be detectable but remain immature by early third postnatal week, as indicated by the insignificant difference in BOLD peak amplitude at P21 compared to the time of eyelid opening at P14 in the current study. The marked increase in visual responsiveness after the third postnatal week appeared to be consistent with a previous study on functional postnatal development of visual evoked potentials in the rat primary visual cortex, when the critical period was found to begin by the end of the third postnatal week, and peak between the fourth and fifth week [7]. While the changes in cerebral blood flow were believed to be associated with changes in synaptic activity [8], a large proliferation of synapse formation by intrinsic neurons was found to occur from P25 to between P30 and P40, but less between the time of eyelid opening and P25 $[9,10]$. The mRNA expression of cpg15, an activity-regulated gene that coordinately regulates the maturation of synapses increased progressively in the SC from postnatal week 2 to 5 [11], whereby dendritic growth was shown to continue beyond P30 under the influence of the optic input [9, 12]. Our experimental approach can be potentially useful in establishing the links between where and when maturational changes occur in the brain, and how these changes are related to the visual sensory development. In particular, while there are $52 \%$ of the primate optic nerve fibers crossing the optic chiasm to the contralateral posterior visual components, more than $98.5 \%$ of the retinal ganglion cell axons in rats decussate to the contralateral superior colliculus at the optic chiasm [13]. Functional MRI in the developmental rat model of visual functions can thus be useful in understanding the effects of unilateral alterations in visual maturation as the contralateral posterior visual components receiving input from an experimental eye can be directly and internally compared to the ipsilateral ones receiving input from the control eye of the same animal, minimizing biological variations from between-group comparisons. Several studies have demonstrated the functional impact of visual cortical damage on subcortical target structures in the thalamus and midbrain [14-21]. It has also been shown that the cerebral cortex has the ability to adapt to altered sensory inputs and can undergo massive restructuring of neuronal circuits during functional reorganization [22]. While the present study showed occasional activations of the visual cortex upon the current stimulation and scanning parameters, further experiments will be performed to understand the functional development of the visual cortex in the same rat developmental model, and the functional impairment and restoration in both the superior colliculus and the visual cortex in the well-established rat model of neonatal hypoxic-ischemic cerebral injury [23-28].

\section{CONCLUSIONS}

By studying the BOLD-fMRI measurements upon unilateral flash illumination to the contralateral eye, our experimental findings showed that the regional BOLD visual 
responses in the rat superior colliculus underwent a systematic increase in amplitude with age after the third postnatal week, while between postnatal days 14 and 21 no significant difference in BOLD signal increase was found. The results of the study are important in establishing in a global, longitudinal and non-invasive manner, a relationship between hemodynamic responses and functional plasticity in normally developing brains. This lays a foundation for using fMRI to study visual maturations and alterations in the postnatal rat models.

\section{REFERENCES}

[1] M. T. Colonnese, M. A. Phillips, M. Constantine-Paton, K. Kaila, and A. Jasanoff, "Development of hemodynamic responses and functional connectivity in rat somatosensory cortex," Nat Neurosci, vol. 11, pp. 72-9, Jan 2008.

[2] M. L. Seghier, F. Lazeyras, and P. S. Huppi, "Functional MRI of the newborn," Semin Fetal Neonatal Med, vol. 11, pp. 479-88, Dec 2006.

[3] M. Hoehn, K. Nicolay, C. Franke, and B. van der Sanden, "Application of magnetic resonance to animal models of cerebral ischemia," J Magn Reson Imaging, vol. 14, pp. 491-509, Nov 2001.

[4] G. A. Lodygensky, T. E. Inder, and J. J. Neil, "Application of magnetic resonance imaging in animal models of perinatal hypoxic-ischemic cerebral injury," Int J Dev Neurosci, vol. 26, pp. 13-25, Feb 2008.

[5] C. P. Pawela, A. G. Hudetz, B. D. Ward, M. L. Schulte, R. Li, D. S. Kao, M. C. Mauck, Y. R. Cho, J. Neitz, and J. S. Hyde, "Modeling of region-specific fMRI BOLD neurovascular response functions in rat brain reveals residual differences that correlate with the differences in regional evoked potentials," Neuroimage, vol. 41, pp. 525-34, Jun 2008.

[6] N. Van Camp, M. Verhoye, C. I. De Zeeuw, and A. Van der Linden, "Light stimulus frequency dependence of activity in the rat visual system as studied with high-resolution BOLD fMRI," J Neurophysiol, vol. 95, pp. 3164-70, May 2006.

[7] M. Fagiolini, T. Pizzorusso, N. Berardi, L. Domenici, and L. Maffei, "Functional postnatal development of the rat primary visual cortex and the role of visual experience: dark rearing and monocular deprivation," Vision Res, vol. 34, pp. 709-20, Mar 1994.

[8] N. K. Logothetis, J. Pauls, M. Augath, T. Trinath, and A. Oeltermann, "Neurophysiological investigation of the basis of the fMRI signal," Nature, vol. 412, pp. 150-7, Jul 122001.

[9] R. D. Lund and J. S. Lund, "Modifications of synaptic patterns in the superior colliculus of the rat during development and following deafferentation," Vision Res, vol. Suppl 3, pp. 281-98, 1971.

[10] R. D. Lund and J. S. Lund, "Development of synaptic patterns in the superior colliculus of the rat," Brain Res, vol. 42, pp. 1-20, Jul 131972.

[11] W. C. Lee and E. Nedivi, "Extended plasticity of visual cortex in dark-reared animals may result from prolonged expression of cpg15-like genes," J Neurosci, vol. 22, pp. 1807-15, Mar 12002.

[12] S. S. Warton and D. G. Jones, "Postnatal development of the superficial layers in the rat superior colliculus: a study with Golgi-Cox and Kluver-Barrera techniques," Exp Brain Res, vol. 58, pp. 490-502, 1985.

[13] M. D. Fleming, R. M. Benca, and M. Behan, "Retinal projections to the subcortical visual system in congenic albino and pigmented rats," Neuroscience, vol. 143, pp. 895-904, Dec 2006.

[14] R. J. Rushmore, B. R. Payne, and S. G. Lomber, "Functional impact of primary visual cortex deactivation on subcortical target structures in the thalamus and midbrain," J Comp Neurol, vol. 488, pp. 414-26, Aug 8 2005.

[15] H. Bridge, O. Thomas, S. Jbabdi, and A. Cowey, "Changes in connectivity after visual cortical brain damage underlie altered visual function," Brain, vol. 131, pp. 1433-44, Jun 2008.

[16] T. Ro and R. Rafal, "Visual restoration in cortical blindness: insights from natural and TMS-induced blindsight," Neuropsychol Rehabil, vol. 16, pp. 377-96, Aug 2006.

[17] J. R. Villablanca and D. A. Hovda, "Developmental neuroplasticity in a model of cerebral hemispherectomy and stroke," Neuroscience, vol. 95, pp. 625-37, 2000.
[18] A. Ptito and S. E. Leh, "Neural substrates of blindsight after hemispherectomy," Neuroscientist, vol. 13, pp. 506-18, Oct 2007.

[19] O. Braddick, J. Atkinson, B. Hood, W. Harkness, G. Jackson, and F. Vargha-Khadem, "Possible blindsight in infants lacking one cerebral hemisphere," Nature, vol. 360, pp. 461-3, Dec 31992.

[20] R. Werth, "Cerebral blindness and plasticity of the visual system in children. A review of visual capacities in patients with occipital lesions, hemispherectomy or hydranencephaly," Restor Neurol Neurosci, vol. 26, pp. 377-89, 2008.

[21] P. Eken, L. S. de Vries, O. van Nieuwenhuizen, N. E. Schalij-Delfos, D. Reits, and H. Spekreijse, "Early predictors of cerebral visual impairment in infants with cystic leukomalacia," Neuropediatrics, vol. 27, pp. 16-25, Feb 1996.

[22] T. Keck, T. D. Mrsic-Flogel, M. Vaz Afonso, U. T. Eysel, T. Bonhoeffer, and M. Hubener, "Massive restructuring of neuronal circuits during functional reorganization of adult visual cortex," Nat Neurosci, vol. 11, pp. 1162-7, Oct 2008.

[23] J. Yang, P. L. Khong, Y. Wang, A. C. Chu, S. L. Ho, P. T. Cheung, and E. X. Wu, "Manganese-enhanced MRI detection of neurodegeneration in neonatal hypoxic-ischemic cerebral injury," Magn Reson Med, vol. 59, pp. 1329-39, Jun 2008.

[24] J. Yang and E. X. Wu, "Detection of cortical gray matter lesion in the late phase of mild hypoxic-ischemic injury by manganese-enhanced MRI," Neuroimage, vol. 39, pp. 669-79, Jan 152008.

[25] K. C. Chan, P. L. Khong, H. F. Lau, P. T. Cheung, and E. X. Wu, "Late measures of microstructural alterations in severe neonatal hypoxic-ischemic encephalopathy by MR diffusion tensor imaging," Int J Dev Neurosci, vol. (In-press), 2009.

[26] S. Wang, E. X. Wu, C. N. Tam, H. F. Lau, P. T. Cheung, and P. L. Khong, "Characterization of white matter injury in a hypoxic-ischemic neonatal rat model by diffusion tensor MRI," Stroke, vol. 39, pp. 2348-53, Aug 2008.

[27] Y. Wang, P. T. Cheung, G. X. Shen, I. Bhatia, E. X. Wu, D. Qiu, and P. L. Khong, "Comparing diffusion-weighted and T2-weighted MR imaging for the quantification of infarct size in a neonatal rat hypoxic-ischemic model at 24 h post-injury," Int J Dev Neurosci, vol. 25, pp. 1-5, Feb 2007.

[28] Y. Wang, P. T. Cheung, G. X. Shen, E. X. Wu, G. Cao, I. Bart, W. H. Wong, and P. L. Khong, "Hypoxic-ischemic brain injury in the neonatal rat model: relationship between lesion size at early MR imaging and irreversible infarction," AJNR Am J Neuroradiol, vol. 27, pp. 51-4, Jan 2006. 